\title{
The Incidence, Risk Factors and Common Foetal outcome of Chorioamnionitis in Women with Preterm Premature Rupture of Membrane (PPROM): A Single Centre Study
}

\author{
NILUFAR SHAMEEM AFZA ${ }^{1}$, WAHEEDA NARGIS ${ }^{2}$, BORHAN UDDIN AHAMED ${ }^{3}$, NAZMUL HAQ SIKDER ${ }^{4}$
}

\begin{abstract}
:
Background and objective: The objective of this study was to detect the incidence of chorioamnionitis in PPROM and to evaluate the pattern of foetal outcome in these cases at a tertiary care hospital in Bangladesh. This study is also aimed to analyze the influence of the demographic variables predisposing to chorioamnionitis in PPROM in a locality. Materials and

Methods: This single centre cross-sectional study was conducted on 110 pregnant women with preterm rupture of membranes at a tertiary care hospital in Dhaka, Bangladesh. The study group was divided according to the presence or absence of clinical chorioamnionitis defined as the presence of two or more of the following criteria: maternal temperature $>38^{\circ} \mathrm{C}$ on two or more occasions ${ }^{3} 1 \mathrm{~h}$ apart, maternal tachycardia ( ${ }^{3} 120$ beats $\left./ \mathrm{min}\right)$, uterine tenderness, foul smelling amniotic fluid, maternal leukocytosis ${ }^{3} 20,000 \mathrm{~mm}^{-3}$ with bands and positive $C$ reactive protein. Antibiotics and tocolysis were used according to the hospital protocols. Foetal outcome was measured on the basis of weight of the baby, and presence of infection (fever), Apgar score and neonatal death. Analysis was performed using SPSS-12.

Results: In this study the rate of chorioamnionitis in PPROM was 25.4\%. The chorioamnionitis was found to be frequent (57.1\%) in younger age group between 15-25 years while PPROM without chorioamnionitis was common (53.6\%) among the age group between 26-35 years. Lower socioeconomic class and history of previous PROM and D\&C was commonly associated with chorioamnionitis though statistically insignificant $(\mathrm{p}>0.05)$. There were also no significant differences in mean birth weight, Apgar scores at 1 and 5 min, rates of respiratory distress syndrome, between patients with and without clinical chorioamnionitis. However, Majority of the babies born to mother with chorioamnionitis showed higher degree of very low birth weight and foetal infection. Neonatal deaths was also higher in the PPROM with chorioamnionitis as compared to the other (32.1\% verses $24.3 \%$ ), though statistically insignificant. In cases of clinical chorioamnionitis the neonates stayed longer in the neonatal intensive care unit (NICU).

Conclusion: The risk of chorioamnionitis in PPROM is relatively higher in our locality. Adverse foetal outcome is more frequent in patients with clinical chorioamnionitis in PPROM. Strategies should be developed to reduce the incidence of chorioamnionitis in PPROM and to ensure safer foetal outcomes in such cases. Regular prenatal care may prove as an effective tool in identification of high risk groups in this regard.
\end{abstract}

Keywords: PPROM, Chorioamnionitis, fetal outcome.

\section{Introduction:}

Premature rupture of the membranes (PROM), defined as rupture of the chorioamniotic membranes before the onset of labor, is a very common clinical problem in human pregnancy. In clinical obstetric terminology,
PROM is defined as rupture of the membranes at term (within 3 weeks of the Estimated Date of Confinement, or due date). The reported incidence of PROM in term pregnancy is $8-10 \%$. Approximately one-fourth of PROM cases occur remote from term (i.e., at less

1. Associate Professor, Department of Gynae \& Obst., Uttara Adhunik Medical College, Uttara Model Town, Dhaka.

2. Associate Professor, Department of Biochemistry, Uttara Adhunik Medical College,

3. Assistant Registrar, Department of Surgery, Uttara Adhunik Medical College

4. Senior Consultant, Northern Medical Colleg \& Hospital 
than 37 completed gestational weeks) and are termed preterm PROM (often called PPROM) ${ }^{1}$. It is responsible for $35 \%$ of all preterm delivery 2 . Recurrence of PROM may occur in $20 \%$ cases. $^{3}$

When PROM occurs earlier from term, there are significant risks of maternal and perinatal morbidity and mortality; therefore the attending physicians play an important role in the management of PPROM ${ }^{1}$. The rate of maternal complications in preterm PROM with chorioamnionitis is as high as $25-35 \%{ }^{3}$. Research in last decade has shown Chorioamnionitis as one of the most common maternal complications (13-60\%) of all associated with preterm PROM which again itself can cause serous consequences for both mother and fetus ${ }^{4}$. It has also been demonstrated that women with PPROM have a higher incidence of positive amniotic fluid culture obtained by amniocentesis even when there is no clinical doubt for chorioamnionitis. ${ }^{5}$

Chorioamnionitis is an infection of the amniotic fluid and its surrounding membrane (or placental tissues) that occurs before, during, or immediately after (within 24 hours) birth. It occurs in approximately 1-5\% of term pregnancies and in as many as $25 \%$ of preterm deliveries. ${ }^{5,6}$ This condition is associated with a higher frequency of cesarean delivery, prolonged maternal febrile morbidity, and an increased rate of neonatal infection, all of which result in prolonged hospitalization and expense for mother and neonate. Several important risk factors for chorioamnionitis have been identified, including the length of labor, duration of rupture of membranes (ROM), duration of internal monitoring, socioeconomic status of patient, preexisting genital-tract infection, and the number of vaginal examinations ${ }^{6,7}$.

The management of the patients with PPROM remains a dilemma for the obstetricians. To date no strategies have been identified that reduce the occurrence of preterm birth after PPROM, thus most pregnancies complicated by PPROM end in preterm birth ${ }^{8}$. Even with conservative management, $50-60 \%$ of women with preterm $\mathrm{PROM}$ remote from term will deliver within 1 week of membrane rupture. However, clinical chorioamnionitis is an independent indication for induction of labor. But there are controversies about the benefits/risks of delaying labor in such critical issue; especially concerning the foetal outcome ${ }^{4}$.

The frequency and severity of neonatal complications following PPROM is determined by a number of maternal factors; most prevalent indicator is chorioamnionitis. A number of studies done in different countries have related chorioamnionitis to different foetal outcome at variable extent. But, no report is available on such study to be conducted on the population of Bangladesh. For this reason, this study was undertaken to detect the incidence of chorioamnionitis in PPROM and to evaluate the pattern of foetal outcome in these cases at a tertiary care hospital in Bangladesh.

\section{Aim and objective:}

This study was aimed

1. To see the frequency of chorioamnionitis in a tertiary care hospital.

2. To study the socio-economic factors of the patients in a locality.

3. To study the presentation of maternal factors in cases with chorioamnionitis in PPROM.

4. To detect the neonatal complications in cases with chorioamnionitis in PPROM.

5. To analyze the pattern of foetal outcome in these cases.

\section{Materials and Methods:}

This cross sectional study was carried out between January 2008 and June 2009 in the department of Gynecology and Obstetrics at Uttara Adhunik Medical College \& Hospital (UAMC\&H), a tertiary care hospital in Dhaka, Bangladesh. During this period of 1 and a half year, a total of 116 pregnant women admitted for PPROM at the Obstetrics department were selected for the study. Among them about 110 women with spontaneous rupture of the membrane between the gestational age of 28 and 36 completed weeks, who willingly consented to participate, were included in this study. Pregnancies before 28 weeks of gestation, women with established labor, multiple pregnancy and any complication other than PPROM that affects foetal and neonatal outcome, e.g., IUGR, diabetes, foetal malformation, pre-eclampsia were excluded.

During admission, patients' demographic variable and complete obstetric history were recorded in a proforma. The diagnosis of ruptured membranes was made by history of a gush of fluid from the vagina, observation of vaginal pooling on speculum evaluation and demonstration of alkaline $\mathrm{pH}$ of vaginal fluid by litmus paper. During speculum examination high vaginal swab was collected for culture and sensitivity and cervical dilatation and effacement was assessed at the same time. Gestational age was determined from LMP and from early USG scan. Routine vital signs were obtained 
and physical examination was performed. Electronic fetal heart rate and tocodynamometer monitoring was used to detect fetal distress and uterine activity.

Plan of management was decided on gestational age, cervical condition, latent period, presentation of the fetus, symptoms and signs of infection. Initially, all patients received a single course of dexamethasone consisting of two $12.5 \mathrm{mg} \mathrm{I/M}$ injection 12 hourly after admission. Preterm labor was defined as regular uterine contractions associated with cervical change or an initial cervical examination of e" $2 \mathrm{~cm}$ or $80 \%$ effacement. After an observation period of $12 \mathrm{~h}$, patients without evidence of labor, fetal distress or infection were accepted in latency period and managed expectantly. Short term tocolysis was given in patients who showed uterine contraction in order to allow steroid therapy to produce maximal effect on pulmonary maturation.

Besides, all patients were managed conservatively with bed rest and with bathroom privileges. During this period the patients were advised to wear a sterile pad which was inspected every four hourly to detect any change of color of liquor and also to document amount of loss. If patient developed signs and symptoms of infection or conservative approach failed then pregnancy was terminated by induction, augmentation or caesarian section. Physical examination was performed daily and temperature was taken four times a day. Bimanual pelvic examinations were avoided unless the patient was accepted to be active labor or a decision to induce labor had been made. Fetal surveillance was checked by daily fetal kick count and auscultation of fetal heart sound 4 hourly. All patients received prophylactic antibiotic for 7 days after admission. Inj. Ampicillin / Cephradin $500 \mathrm{mg}$ I/V 6 hourly for 48 hours, then this regimen was changed to oral form. This antibiotic was continued for seven days if patient remain undelivered.

Indications for delivery included cervical dilatation were $4 \mathrm{~cm}$ and $80 \%$ effacement despite to the tocolytic treatment, clinical diagnosis of chorioamnionitis, hemorrhage, and fetal distress. The labor was induced with misoprostol or augmented with oxytocin drip if there was no contraindication or underwent caesarean section. Cesarean section was performed for the usual obstetric indications.

The diagnosis of clinical chorioamnionitis was made in the presence of two or more of the following criteria: maternal temperature $>37.8^{\circ} \mathrm{C}$ on two or more occasions e" $1 \mathrm{~h}$ apart, maternal tachycardia (e"120 beats/min), fetal tachycardia (e"160 beats/min), uterine tenderness, foul smelling amniotic fluid, maternal leukocytosis e" $20,000 / \mathrm{mm}^{3}$ with bands and positive C reactive protein ${ }^{9}$. All patients with clinical chorioamnionitis were given broad spectrum antibiotics parenterally and labor was induced. The antibiotic therapy was maintained during labor. Antibiotic was also given to the baby after delivery in such cases. All the neonates were referred to neonatal ward for further management according to the hospital protocol.

Data collected after delivery included 1- and 5-min Apgar scores, birth weight, route of delivery, placental cultures and results of neonatal outcome including RDS which was defined by the presence of characteristic radiographic findings and an oxygen requirement at $24 \mathrm{~h}$. From the primary data obtained, tables were made and interpreted. Data are presented as incidence (\%) or mean \pm SD. All data was checked and edited after collection. Data was applied in the SPSS 12 for statistical analysis.

\section{Results:}

The total number of deliveries conducted at the hospital during study period was 1450, out of this $116(8 \%)$ women presented with PPROM, of which 110 cases participated in this study. Among them 28 (25.4\%) cases were finally detected with chorioamnionitis prior to delivery (figure-1).

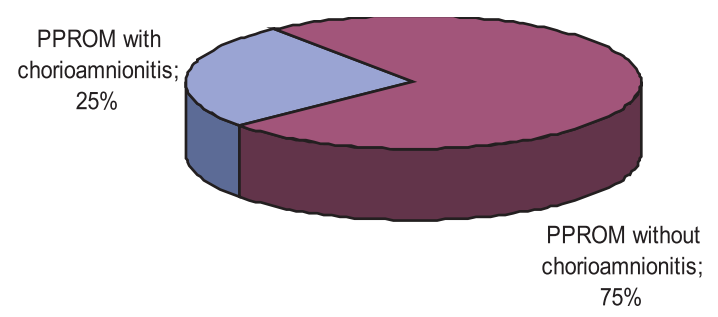

Fig.-1: Frequency of chorioamnionitis in PPROM

The mean maternal age was $28.62 \pm 7.4$ years ranging from 16 to 40 years. Table-I shows the distribution of the study subjects according to age where 16 (57.1\%) cases of PPROM with chorioamnionitis are between 16 - 25 years and 44(53.6\%) cases without chorioamnionitis are within $26-35$ years. However, the mean age of the two groups showed no significant difference $(p>0.05)$.

Table - II shows the relevant maternal demographic characteristics of the women in PPROM with and without chorioamnionitis. PPROM is extremely influenced by the socio-economic status or standard of living (SLI: standard of living index) which, in this study, is determined by an individual's monthly income, educational background, occupation, 
Table-I

Distribution of the study subjects according to age

\begin{tabular}{lccc}
\hline Maternal Age & $\begin{array}{c}\text { PPROM with } \\
\text { chorioamnionitis }(\mathrm{n}=28)\end{array}$ & $\begin{array}{c}\text { PPROM without } \\
\text { chorioamnionitis }(\mathrm{n}=82)\end{array}$ & Total $(\mathrm{n}=110)$ \\
\hline $16-25$ years & $16(57.1 \%)$ & $25(30.4 \%)$ & $41(37.2 \%)$ \\
$26-35$ years & $12(42.8 \%)$ & $44(53.6 \%)$ & $56(50.9 \%)$ \\
$36-40$ years & 0 & $13(15.8 \%)$ & $13(11.8 \%)$ \\
Mean age & $26.43 \pm 4.1$ years & $30.81 \pm 3.1$ years & $\mathrm{p}>0.05$ \\
\hline
\end{tabular}

Table-II

Distribution of the study subjects according to maternal demographic Variables

\begin{tabular}{|c|c|c|c|}
\hline Variables & $\begin{array}{c}\text { PPROM with } \\
\text { chorioamnionitis( } \\
n=28)\end{array}$ & $\begin{array}{c}\text { PPROM } \\
\text { without } \\
\text { chorioamnionitis } \\
(\mathrm{n}=82) \\
\end{array}$ & $\begin{array}{c}\text { Total } \\
(n=110)\end{array}$ \\
\hline \multicolumn{4}{|l|}{ Monthly income: } \\
\hline$<4000$ taka & $16(57.1 \%)$ & $10(12.1 \%)$ & $26(23.6 \%)$ \\
\hline 4000 to 8000 taka & $8(28.5 \%)$ & $23(28 \%)$ & $31(28.1 \%)$ \\
\hline$>8000$ taka & $4(14.2 \%)$ & $49(59.7 \%)$ & $53(48.1 \%)$ \\
\hline \multicolumn{4}{|l|}{ Level of Education: } \\
\hline Illiterate & $3(10.7 \%)$ & $4(4.8 \%)$ & $7(6.3 \%)$ \\
\hline Up to Primary & $6(21.4 \%)$ & $7(8.5 \%)$ & $13(11.8 \%)$ \\
\hline Secondary (up to SSC or above primary) & $13(46.4 \%)$ & $34(41.4 \%)$ & $47(42.7 \%)$ \\
\hline Higher Secondary (up to HSC or above SSC) & $6(21.4 \%)$ & $37(45.1 \%)$ & $43(39 \%)$ \\
\hline \multicolumn{4}{|l|}{ Residence: } \\
\hline Urban & $15(53.5 \%)$ & $57(69.5 \%)$ & $72(65.4 \%)$ \\
\hline Slum & $9(32.1 \%)$ & $3(3.6 \%)$ & $12(10.9 \%)$ \\
\hline Rural & $4(14.2 \%)$ & $22(26.8 \%)$ & $26(23.6 \%)$ \\
\hline \multicolumn{4}{|l|}{ Occupation: } \\
\hline House-wife/ unemployed & $5(17.8 \%)$ & $15(18.2 \%)$ & $20(18.1 \%)$ \\
\hline Day laborers/Garments worker/ Other & $17(60.7 \%)$ & $25(30.4 \%)$ & $42(38.1 \%)$ \\
\hline Service holder & $6(21.4 \%)$ & $42(51.2 \%)$ & $48(43.6 \%)$ \\
\hline \multicolumn{4}{|l|}{ Standard of living: } \\
\hline Low & $15(53.5 \%)$ & $24(29.2 \%)$ & $39(35.4 \%)$ \\
\hline Medium & $8(28.5 \%)$ & $47(57.3 \%)$ & $55(50 \%)$ \\
\hline high & $5(17.8 \%)$ & $11(13.4 \%)$ & $16(19.5 \%)$ \\
\hline
\end{tabular}

housing, personal hygiene, nutritional status, sanitation, source of water, and co-existing infectious or nutritional disorders ${ }^{10,11}$. According to the SLI, 55 (50\%) study subjects of all showed medium standard of living. $15(53.5 \%)$ cases with clinical chorioamnionitis belonged to lower socio-economic group whereas 47 (57.3\%) women in PPROM without chorioamnionitis were found to have medium standard of living (figure-2). The differences of socio-economic status between the two groups of PPROM was statistically insignificant ( $p>0.05)$.

Table - III shows the maternal obstetric characteristics of the women in PPROM with and without chorioamnionitis. The mean gestational age on

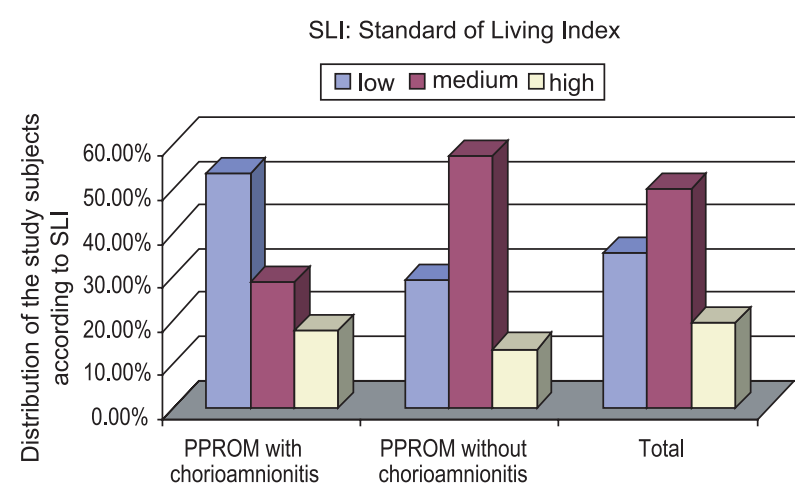

Fig.-2: Distribution of the study subjects according to their standard of living 
Table-III

Distribution of the study subjects according to maternal obstetric characteristics.

\begin{tabular}{lccc}
\hline Obstetric variables & $\begin{array}{c}\text { PPROM with } \\
\text { chorioamnionitis } \\
(\mathrm{n}=28)\end{array}$ & $\begin{array}{c}\text { PPROM without } \\
\text { chorioamnionitis } \\
(\mathrm{n}=82)\end{array}$ & $\mathrm{p}$ \\
\hline Mean Gestational age at PPROM (weeks) & $30.2 \pm 3.2$ & $31.3 \pm 3.7$ & 0.543 \\
Mean Gestational age at delivery (weeks) & $31.2 \pm 3.4$ & $31.7 \pm 3.5$ & 0.622 \\
Latency period (hours) & $60 \pm 53$ & $75 \pm 104$ & 0.104 \\
Mean prolongation of pregnancy (days) & $3 \pm 2.0$ & $4 \pm 5.0$ & 0.584 \\
Parity : & & & 0.534 \\
$\quad$ Nulliparous & $15(53.5 \%)$ & $36(43.9 \%)$ & \\
$\quad$ Multiparous & $13(46.4 \%)$ & $46(56 \%)$ & 0.689 \\
Antenatal care: & & & \\
$\quad$ Regular & $3(10.7 \%)$ & $17(20.7 \%)$ & \\
$\quad$ Irregular & $11(39.2 \%)$ & $33(40.2 \%)$ & \\
$\quad$ None & $14(50 \%)$ & $32(39 \%)$ & \\
\hline
\end{tabular}

admission was $30.7 \pm 3.4$ weeks; the mean gestational age at delivery was $31.4 \pm 3.4$ weeks. There were no significant differences in mean gestational age at PPROM and at delivery, latency period, mean prolongation of pregnancy, parity, and antenatal care between patients with and without clinical chorioamnionitis ( $p>0.05)$. However, majority (53.5\%) of the women with clinical chorioamnionitis were nulliparous compared to the women without chorioamnionitis who were mostly (56\%) multiparous. Antenatal or prenatal care was not reported in 50\% and $39 \%$ of the women with and without chorioamnionitis, respectively. Out of all study subjects of PPROM, only 20 (18.1\%) had regular and $44(40 \%)$ irregular checkups antenatally. Among them, $39.2 \%$ chorioamnionitis case and $40.2 \%$ nochorioamnionitis case reported of irregular antenatal checkups (figure-3).

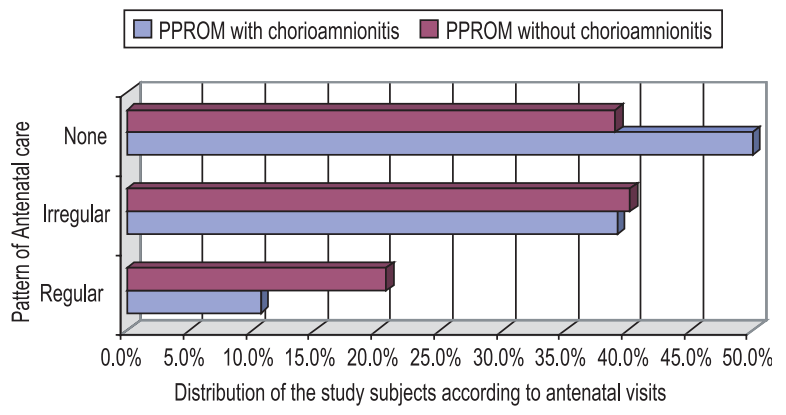

Fig.-3: Distribution of the study subjects according to maternal antenatal check-up.
The distribution of major health factors or diseases related to maternal health in current pregnancy and the relevant past obstetric history is displayed in TableIV. Chorioamnionitis increased significantly with maternal anemia (at $O R=8.9$ ). Urinary tract infection (UTI) and the lower genital tract infections were more (39.2\% and $53.5 \%$, respectively) frequent in cases with chorioamnionitis than the no-chorioamnionitis mothers (29.2\% and $30.4 \%)$, though statistically not significant. Of all the study subjects, $45.4 \%$ had no history of sexual activity including majority (56.1\%) of the women without chorioamnionitis. However, 57.1\% cases with chorioamnionitis gave history of coitus within 1 month and $17.8 \%$ within 2 days. There was no significant difference regarding the history of abortion, PROM, preterm delivery following PROM, MR or D\&C between the patients with and without chorioamnionitis $(p>0.05)$. However, majority $(21.4 \%)$ of the women with chorioamnionitis reported of previous PROM and D\&C; though the rates were higher in no-chorioamnionitis PPROM mothers.

Table $-V$ shows the distribution of common foetal outcome in PPROM with and without chorioamnionitis. The rate of cesarean section was $60.9 \%$. Surgical indications included malpresentation (9.4\%), fetal distress (22.7\%), repeat cesarean section (28.3\%) and prematurity (39.6\%). There was no statistical difference in the rate of cesarean delivery among the women with and without chorioamnionitis $(p>0.05)$. The fetal sex rate was 62 (56.3\%) male to 48 (43.6\%) female. The neonatal mortality rate was $26.3 \%$ with higher rates (32.1\%) confined to the chorioamnionitis 
Table- IV

Distribution of the study subjects according to associated maternal factors / diseases.

\begin{tabular}{lccc}
\hline Variables & $\begin{array}{c}\text { PPROM with } \\
\text { chorioamnionitis } \\
(\mathrm{n}=28)\end{array}$ & $\begin{array}{c}\text { PPROM without } \\
\text { chorioamnionitis } \\
(\mathrm{n}=82)\end{array}$ & $\begin{array}{c}\text { Total } \\
(\mathrm{n}=110)\end{array}$ \\
\hline Anemia & $23(82.1 \%)$ & $26(31.7 \%)$ & $49(44.5 \%)$ \\
Urinary tract infection (UTI) & $11(39.2 \%)$ & $24(29.2 \%)$ & $35(31.8 \%)$ \\
Lower genital tract infection & $15(53.5 \%)$ & $25(30.4 \%)$ & $40(36.3 \%)$ \\
Time of last coitus: & & & \\
$\quad$ within 48 hours & $5(17.8 \%)$ & $10(12.1 \%)$ & $15(13.6 \%)$ \\
$\quad 2-7$ days & $3(10.7 \%)$ & $15(18.2 \%)$ & $18(16.3 \%)$ \\
$\quad 7$ days to 1 month & $16(57.1 \%)$ & $11(13.4 \%)$ & $27(24.5 \%)$ \\
$\quad$ None & $4(14.2 \%)$ & $46(56.1 \%)$ & $50(45.4 \%)$ \\
Relevant past history: & $1(3.5 \%)$ & $13(15.8 \%)$ & $14(12.7 \%)$ \\
$\quad$ Abortion & $6(21.4 \%)$ & $22(26.8 \%)$ & $28(25.4 \%)$ \\
PROM & $2(7.1 \%)$ & $12(14.6 \%)$ & $14(12.7 \%)$ \\
Preterm delivery due to PROM & $3(10.7 \%)$ & $13(15.8 \%)$ & $16(14.5 \%)$ \\
$\quad$ MR & $6(21.4 \%)$ & $10(12.1 \%)$ & $16(14.5 \%)$ \\
$\quad$ D\&C & & & \\
\hline
\end{tabular}

Table-V

Distribution of the study subjects according to foetal / neonatal outcome

\begin{tabular}{|c|c|c|c|c|}
\hline \multicolumn{2}{|l|}{ Foetal / neonatal outcome } & $\begin{array}{c}\text { PPROM with } \\
\text { chorioamnionitis } \\
(n=28)\end{array}$ & $\begin{array}{l}\text { PPROM without } \\
\text { chorioamnionitis } \\
(\mathrm{n}=82)\end{array}$ & $\begin{array}{c}\text { Total } \\
(n=110)\end{array}$ \\
\hline \multicolumn{2}{|l|}{ Caesarean section } & $18(64.2 \%)$ & $49(59.7 \%)$ & $67(60.9 \%)$ \\
\hline \multirow{5}{*}{$\begin{array}{l}\text { Birth weight: } \\
\text { Very low birth weight: } \\
\text { Low birth weight: }\end{array}$} & Mean (g) & $1,750 \pm 654$ & $2,321 \pm 354$ & $p=0.683$ \\
\hline & $<1.5 \mathrm{Kg}$ & $9(32.1 \%)$ & $3(3.6 \%)$ & $12(10.9 \%)$ \\
\hline & a] $1.5-2.0 \mathrm{Kg}$ & $11(39.2 \%)$ & $32(39 \%)$ & $43(39 \%)$ \\
\hline & b] $2.1-2.4 \mathrm{Kg}$ & $5(17.8 \%)$ & $43(52.4 \%)$ & $48(43.6 \%)$ \\
\hline & $\geq 2.5 \mathrm{Kg}$ & $3(10.7 \%)$ & $4(4.8 \%)$ & $7(6.3 \%)$ \\
\hline \multicolumn{2}{|l|}{ Neonatal sex, male } & $19(67.8 \%)$ & $43(52.4 \%)$ & $62(56.3 \%)$ \\
\hline \multicolumn{5}{|l|}{ Apgar score $\leq 7$ at - } \\
\hline & $1 \mathrm{~min}$ & $18(64.2 \%)$ & $47(57.3 \%)$ & $65(59 \%)$ \\
\hline & $5 \mathrm{~min}$ & $10(35.7 \%)$ & $25(30.4 \%)$ & $35(31.8 \%)$ \\
\hline \multicolumn{2}{|l|}{ Neonatal asphyxia } & $3(10.7 \%)$ & $7(8.5 \%)$ & $10(9.1 \%)$ \\
\hline \multicolumn{2}{|c|}{ Respiratory distress syndrome (RDS)17 (60.7\%) } & $40(48.7 \%)$ & $57(51.8 \%)$ & \\
\hline \multicolumn{2}{|l|}{ Neonatal jaundice } & $6(21.4 \%)$ & $15(18.2 \%)$ & $21(19.1 \%)$ \\
\hline \multicolumn{2}{|l|}{ Neonatal sepsis } & $2(7.1 \%)$ & $5(6.1 \%)$ & $7(6.3 \%)$ \\
\hline \multicolumn{2}{|l|}{ Death } & $9(32.1 \%)$ & $20(24.3 \%)$ & $29(26.3 \%)$ \\
\hline \multicolumn{2}{|c|}{ Mean hospitalization in NICU (days) } & $13 \pm 8$ & $7 \pm 7$ & $p=0.025$ \\
\hline
\end{tabular}

group. There were no difference among the women with and without chorioamnionitis with respect to cesarean delivery rate, Apgar scores d"7 at 1 and 5 min, fetal sex, neonatal mortality, rates of Neonatal asphyxia, RDS, Neonatal jaundice, and Neonatal sepsis; $(p>0.05)$, but a trend towards an increased rate of neonatal adverse outcome was noted in women with chorioamnionitis (figure-4) whereas healthy neonates were reported in $18.2 \%$ cases of PPROM without chorioamnionitis. Moreover, the hospitalization in neonatal intensive care unit (NICU) was also significantly longer in the babies born to the mother with chorioamnionitis $(p<0.05)$. 


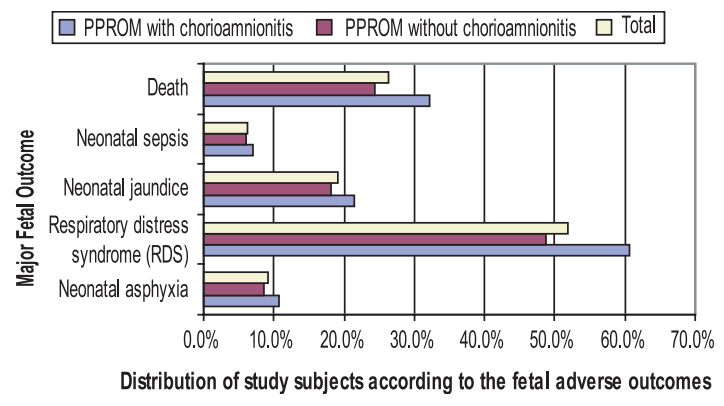

Fig.-4: Distribution of foetal adverse outcome in PPROM with and without chorioamnionitis.

\section{Discussion:}

In this study, the prevalence of PPROM was $8 \%$ of all hospital deliveries which is higher than the reported $2.3 \%$ and $5.4 \%$ by Smith $\mathrm{G}$ et al, ${ }^{12}$ and Tahir $\mathrm{S}$ et al 13 , respectively, but similar rate (8.12\%) was reported in a local study at Dhaka Medical College Hospital, Bangladesh ${ }^{14}$. Clinical chorioamnionitis was observed in $25.4 \%$ women of PPROM and $1.93 \%$ of all the hospital deliveries. This rate is within the ranges published as $13-60 \%{ }^{15,16}$ but is little higher than the observation (14\%) of Akter $\mathrm{S}^{17}$. This high prevalence can be explained by the cultural influences of early marriages, poverty, gender discrimination resulting in low maternal weight gain and lack of birth spacing which are significantly associated with PPROM as has been reported earlier ${ }^{13,14,18}$. In this study younger maternal age, and lower socioeconomic class were more commonly associated with chorioamnionitis in PPROM with higher rates of poverty $(57.1 \%$ with monthly income $<4000 /-)$, illiteracy $(46.4 \% £$ SSC), unemployment and $4^{\text {th }}$ grade employment $(60.7 \%$ of day laborers, garment workers and others); all together leading into lower standard of living (53.5\%). It indicates all the demographic features are interrelated which determine nutrition, living standard, personal hygiene, immunity and awareness of the patient and are responsible for such higher rate of chorioamnionitis in PPROM in our country. On the other hand, higher rates of medium living standard (57.3\%) with grater number of working mother (51.2\%) are facing PPROM without chorioamnionitis despite their better educational background $\left(45.1 \%{ }^{3} \mathrm{HSC}\right)$ and economic solvency (59.7\% with income > 8000/- per month).

The chorioamnionitis is found to be frequent (57.1\%) in younger age group between 15-25 years while PPROM without chorioamnionitis is common (53.6\%) among the age group between $26-35$ years. This indicates that advanced maternal age is protective of chorioamnionitis which is also supported by Aly $\mathrm{H}$ et $\mathrm{al}^{19}$ and Naeye $\mathrm{RL}^{20}$. The mean of gestational week on admission and at delivery are approximately equal. The latency period is also found to be similar in women with and without chorioamnionitis. Finally, the extension of pregnancy for about 3 to 4 days in this study may be attributable to antibiotic chemoprophylaxis which improves the duration of latency after PPROM 12, 16, 21, 22, 23.

In this study the rate of nulliparity $(53.5 \%)$ in women with chorioamnionitis was quite lower than multiparous (56\%) women without chorioamnionitis which is comparable to the findings by Osmanaðaoðlu MA, Ünal S and Bozkaya $\mathrm{H}^{24}$. This could be explained by the fact that nulliparous women generally have longer active phase of labor, increasing the incidence of vaginal examinations and thus raising the probability of introducing pathogenic organisms in utero ${ }^{25}$. Regarding antenatal care, majority (50\%) of the women with chorioamnionitis has no history of antenatal check-up, and among the rest 39.2\% reported of irregular visits. On the other hand, PPROM women with no-chorioamnionitis showed maximum irregular check-ups which accounts for $30 \%$ of all cases and $40.2 \%$ cases of no-chorioamnionitis PPROM. This could be related to the busy metropolitan life style of the majority of the PPROM working mothers which eventually may have attributed in such higher rates of PPROM in middle (57.3\%) and higher (13.4\%) socioeconomic class.

Antenatal check-ups play a significant role in early detection of a number maternal health factors or diseases that may predispose to PPROM. In this study, women with chorioamnionitis have higher rates of anemia, UTI and lower genital tract infections $(82.1 \%, 39.2 \%$ and $53.5 \%)$ where only maternal anemia is statistically significant compared to nochorioamnionitis PPROM mothers $(p<0.05)$. This is consistent with the findings of Aly $\mathrm{H}$ et $\mathrm{al}^{19}$. Anemia and other subclinical infections are associated risk factors of PPROM by affecting nutrition and immunity which again may lead to chorioamnionitis in such cases $^{7,19,26}$. Regarding other associated maternal factors, coitus within 7 days to 1 month is reported in majority $(57.1 \%)$ of the mothers with chorioamnionitis whereas majority (56.1\%) of the no-chorioamnionitis group shows no sexual activity during the current pregnancy. Sexual activities facilitate the entrance of microbial agents into the upper genital tract and 
enhance the rate of chorioamnionitis in PPROM ${ }^{17}$. Previous history of abortion, PROM, preterm delivery following PROM, MR or D\&C was more frequent in women without chorioamnionitis $7,17,19$.

Caesarean section rate for the total population was found to be $60.9 \%$. This is comparable with the result (58.7\%) of Kifas $\mathrm{Al} \mathrm{Qa}^{22}$, but is greater than reported (20\%) by Chales $\mathrm{PJ}^{23}$ and (14\%) by Tahir S et al ${ }^{13}$. Caesarean section, in cases of PPROM with malpresentation and prematurity, is mostly preferred to decrease the chances of traumatic delivery. In this current study, male infants are commonly affected from PPROM (56.3\%), is in agreement with that of Osmanaðaoðlu MA (56\%) $)^{24}$ and Yeo, Tudehope $(31 \%)^{27}$ but is in disagreement with that of Casalaz et al. $(66 \%)^{28}$.

In the current study, the incidence of neonatal complications in women with chorioamnionitis is high. Majority (51.8\%) of the surviving infants had respiratory distress syndrome with $60.7 \%$ babies of born to mothers with chorioamnionitis ${ }^{29}$. Neonatal jaundice (21.4\% compared with $18.2 \%$; odds ratio [OR] $=1.2$, $95 \%$ confidence interval $[\mathrm{Cl}]=0.421$ to $3.524, \mathrm{P}=$ 0.454 ) was the second most frequent outcome observed in this study. Very low birth weight (32.1\% versus 3.6\%) and a low 1 and 5-minute Apgar (64.2\%, $35.7 \%$ compared with $57.3 \%$ and $30.4 \%$ ) occurred more frequently in the chorioamnionitis group; higher rates than the reported $34.8 \%$ by Aziz $\mathrm{N}^{29}$ and $55 \%$ by Ramsey PS ${ }^{30,31}$. Further, $9.1 \%$ of surviving neonates had Neonatal asphyxia; mostly (10.7\%) confined to the chorioamnionitis group. The incidence of neonatal sepsis was $6.3 \%$, a figure that is in line with numbers reported elsewhere (ie, $2 \%$ to $19 \%)^{31}$, 32,33 . The numbers of neonatal complications documented in our study appear to be higher than those provided in previous studies 17, 32, 34, 35; possibly as the effect of higher rates of neonatal survival following delivery at an earlier gestational age.

The most recent Cochrane review of trials of antibiotics in PPROM reported that antibiotics seem to be benefit in the reduction of the numbers of babies requiring neonatal intensive care and ventilation for more than 28 days $^{36}$. In our study, the PPROM to delivery interval between chorioamnionitis present and absent groups was prolonged from 3 to 4 days ( $p>0.05$ ). However, this study did not show evidence of benefit for neonatal outcome especially for reduction of days in hospitalization in NICU. Thus, the neonatal adverse outcome and Hospitalization in NICU was longer in women with chorioamnionitis.

\section{Summary and Conclusion}

This study was carried out to detect the incidence of chorioamnionitis in PPROM at a tertiary level hospital and to evaluate the pattern of foetal outcome in these cases and also to study the pattern of demographic presentation in such cases. For this, 110 cases of PPROM were selected purposively and categorized according to the presence or absence of clinical chorioamnionitis. The demographic features and fetal outcome were monitored among the cases. Foetal outcome was measured on the basis of weight of the baby, and presence of infection (fever), Apgar score and neonatal death.

The higher (25.4\%) incidence of chorioamnionitis in our hospital is acceptable since majority of the cases presented with lower socioeconomic background. Advanced maternal age is protective of chorioamnionitis. The incidence of neonatal complications is high in this study. Thus, there is no difference among the women with and without chorioamnionitis with respect to cesarean delivery rate, Apgar scores d"7 at 1 and 5 min, fetal sex, neonatal mortality, rates of Neonatal asphyxia, RDS, Neonatal jaundice, and Neonatal sepsis; $(p>0.05)$, but a trend towards an increased rate of neonatal adverse outcome and longer hospitalization in neonatal intensive care unit (NICU) are noted in women with chorioamnionitis. Neonatal death is also higher in the PPROM with chorioamnionitis as compared to the other (32.1\% verses $24.3 \%$ ), though statistically insignificant.

PPROM is encountered to be one of the most common clinical events which turn a normal traditional pregnancy suddenly into a high-risk one for both the mother and the fetus/neonate. Chorioamnionitis in PPROM may either exist as a predisposing factor of PPROM or as a serious sequel following PPROM. The epidemi-ological explanatory variables can be explored to screen out the population at higher risk of developing chorioamnionitis with PPROM. Regardless of enormous progress in the therapeutic managements of PPROM, it is still very difficult to halt the adverse fetal outcomes. Thus, more emphasis should be given by the government to ensure early prenatal care among the lower socio-cultural population which remain undermined due to their ignorance and unawareness of the fact. Lastly, this is a single 
centered study based on the patients attending a tertiary reference hospital. To determine the exact incidence and prevalence of chorioamnionitis further large scale studies are required. Such studies may also be beneficial in evaluating the efficacy of early prenatal care to control the incidence of chorioamnionitis.

\section{Acknoledgement:}

The authors would like to thank and deeply acknowledge all the doctors and the other supporting stuff of the Department of Obst \& Gynae at Uttara Adhunik Medical College \& Hospital, Uttara, Dhaka for their kind cooperation during the study.

\section{References:}

1. Johnson JWC, Daikoku NH, Niebyl JR, Johnson TRB, Khouzami VA, Witter FR. Premature rupture of the membranes and prolonged latency. Obstet Gynecol 1981; 57:547-556.

2. Willium's Obstetric Gynaecologic: Pre term labor, Pre labor rupture of the membranes, 19th edition, 1993; Appleton \& Lange, California,USA; p361.

3. Griff Jones; Pre labor rupture of the membrane; Obstetrics \&Gynaecology-An evidenced based text for MRCOG; Arnold, Member of the hodder headline group.www.arnold publishers.com; 1st edition; Oxford University press; 2004; p297.

4. Brian M. Mercer. Preterm Premature Rupture of the Membranes .Obstet Gynecol 2003; 101:178 $-93$.

5. Mercer BM. Premature rupture of the membrane. In: Petraglia F, Strauss GF, Gabbe SG, Wises G. Complicated Pregnancy. 4th ed. London: informa health care; 2007; 713-727.

6. Andrea C Scharfe, Jude P Crino: Pre term labor and Pre labor rupture of the membranes; The Johns Hopkins Manual of Gynecology Obstetrics; 2nd edition, 2000; Lippincot Williams \& Wilkins, USA, p123.

7. Newton E. Chorioamnionitis and intra amniotic infection. Clin Obstet Gynecol. 1993; 36:795808.

8. Denney MJ, Cuhane FJ, Goldenberg LR Prevention of preterm birth. Women's Health 2008; 4:625-38.
9. Gibbs RS, Blanco JD, St Clair PJ, Castaneda YS (1982) Quantitative bacteriology of amniotic fluid from women with clinical intraamniotic infection at term. J Infect Dis 145:1-8.

10. Myrianthopouplos NC, French KS, An Application Of The U.S. Bureau Of The Census Socioeconomic Index To A Large, Diversified Patient Population. Soc Sci Med, 1968; 2, 283.

11. International Institute for Population Sciences. National Family Health Survey (NFHS-2) 199899. Mumbai, India: IIPS, 2000.

12. Smith G, Rafuse C, Anand N, Brenanan B, Connors G, Crane J, et al. Prevalence, Management and outcomes of preterm prelabour rupture of the membranes of women in Canada. J Obstet Gynecol Can 2005;27: 547-53.

13. Tahir S, Aleem M, Aziz R. Incidence and out come of preterm premature rupture of membranes. Pak J Med Sci 2002;18(1):26-32.

14. Tasnim S, A B Bhuiyan: Outcome of premature rupture of membranes; Bangladesh Journal of Obstetrics\& Gynaecology , 1998;13(1),16-20.

15. Holzman $\mathrm{C}$, Lin $\mathrm{X}$, Senagore $\mathrm{P}$, Chung $\mathrm{H}$. Histologic chorioamnionitis and preterm delivery. Am J Epidemiol. 2007;166(7):786-94.

16. Obi SN, Ozumba BC. Pre-term premature rupture of fetal membranes: the dilemma of management in a developing nation. J Obstet Gynaecol. 2007;27(1):37-40.

17. Akter $\mathrm{S}$, Akther R, Rashid M, Preterm Prelabour Rupture of the Membrane \& Feto-Maternal out come: an Observational Study. J Bangladesh Coll Phys Surg 2010; 28: 17-23.

18. Savitz DA, Blackmore CA, Thorp JM; Epidemiologic characteristics of preterm delivery: Etiologic heterogeneity. Am J Obstet Gynaecol 1991; 164: 467-71.

19. Aly $H$, Alhabashi $G$, Hammad TA, Owusu-Ansah S, Bathgate S, Mohamed M, ABO Phenotype and Other Risk Factors Associated with Chorioamnionitis. The Journal of Pediatrics.2008;153(1):16-18.

20. Naeye RL, Maternal Age, Obstetric Complications, and the Outcome of Pregnancy. Obstetrics \& Gynecology, 1983; 61(2): 210-216. 
21. Kifah AL Qa Qa, Awaysheh FA. Neonatal outcome and Prenatal Antibiotics Treatment in Premature Rupture of membranes. Pak J Med Science 2005; 21:441-4.

22. Charles PJ, Muriel R, Charles PJ, Rene E, Olivien C, Pascal G, et al. A prospective population based study of 598 causes of PROM between 24 and 34 weeks gestation description management and morality (Dominos Cohort). Eur J Obstet Gyreacol Reprod Boil 2005; 121:16470.

23. Allen SR . Tocolytic therapy in preterm PROM. Clin Obstet Gynecol 1998; 41:842-848.

24. Osmanaðaoðlu MA, Ünal S, Bozkaya H. Chorioamnionitis risk and neonatal outcome in preterm premature rupture of membranes. Arch of Gynecology And Obstetrics 2005; 271 (1): 3339.

25. Seaward PG, Hannah ME, Myhr TL, Farine D, Ohlsson A, Wang EE, Haque K, Weston JA, Hewson SA, Ohel G, Hodnett ED. International multicenter term prelabor rupture of membranes study: evaluation of predictors of clinical chorioamnionitis and postpartum fever in patients with prelabor rupture of membranes at term. Am J Obstet Gynecol 1997,177: 1024- 1029.

26. Newton ER. Preterm labor, preterm premature rupture of membranes, and chorioamnionitis. Clin Perinatol.2005; 32:571-600.

27. Yeo CL, Tudehope DI (1994) Outcome of resuscitated apparently stillborn infants: a ten year review. J Paediatr Child Health 30:129-133.

28. Casalaz DM, Marlow N, Speidel BD. Outcome of resuscitation following unexpected apparent stillbirth. Arch Dis Child Fetal Neonatal. 1998; 78:112-115
29. Aziz N; Cheng YW; Caughey AB Neonatal outcomes in the setting of preterm premature rupture of membranes complicated by chorioamnionitis. J Matern Fetal Neonatal Med. 2009; 22(9):780-4.

30. Ramsey PS; Lieman JM; Brumfield CG; Carlo W. Chorioamnionitis increases neonatal morbidity in pregnancies complicated by preterm premature rupture of membranes. Am J Obstet Gynecol. 2005; 192(4):1162-6.

31. Botet F; Figueras J; Carbonell-Estrany X; Arca $\mathrm{G}$; Effect of maternal clinical chorioamnionitis on neonatal morbidity in very-low birthweight infants: a case-control study. J Perinat Med. 2010; 38(3):269-73.

32. Elimian A, Verma $U$, Beneck D, Cipriano R, Visintainer $P$, Tejani $N$. Histologic chorioamnionitis, antenatal steroids and perinatal outcomes. Obstet Gynecol.2000; 96:333-336.

33. Soraisham AS, Singhal N, McMillan DD, et al. A multicenter study on the clinical outcome of chorioamnionitis in preterm infants. Am J Obstet Gynecol 2009;200: 372.

34. Dexter SC, Pinar H, Malee MP, Hogan J, Carpenter MW, Vohr BR. Outcome of very low birth weight infants with histopathologic chorioamnionitis. Obstet Gynecol.2000; 96:172 -177 .

35. Vermillion ST, Soper DE, Chasedunn-Roark J. Neonatal sepsis after betamethasone administration to patients with preterm premature rupture of membranes. Am J Obstet Gynecol.1999; 181:320-327.

36. Kenyon S, Boulvain M. Antibiotics for preterm premature rupture of membranes. Cochrane Database Syst Rev.2000; 2: CD001058. 